\title{
New Ways of Revealing Cepheid Binaries
}

\author{
L. Szabados \\ Konkoly Observatory, Hungary
}

\begin{abstract}
Two methods involving the observed amplitudes of radial velocity and $U B V R$ light variations for classical Cepheids have been analysed, both being implicitly known: their principle is trivial but these methods had not yet been used systematically as indicators of duplicity.

The slope method is based on the alteration of the wavelength dependence of the light variation amplitude if either a blue or a red companion is added to the light of the Cepheid. The amplitude ratio (AR) method makes use of the fact that the companion reduces the amplitude of the light variation without observable effect on the pulsational radial velocity amplitude. This means that the ratio of these two amplitudes $\left(A_{\text {rad.vel. }} / A_{B}\right)$ has a larger value for binary Cepheids as compared with the single pulsators.

Each method has been applied to more than 100 Cepheids, thus allowing to study how the uncontaminated parameters (amplitude ratio and slope) depend on the pulsation period. Binary Cepheids deviate from the regular pattern in these diagrams, and a number of new binaries can be discovered in this way. The effect of duplicity is revealed by both methods independently for VZ CMa, FM Cas, CR Cep, V402 Cyg, V1154 Cyg, V440 Per and DR Vel.
\end{abstract}

\section{The "slope"-method}

A new treatment of the amplitude ratios is suggested which makes use of four photometric amplitudes simultaneously. The relationship between the photometric amplitudes in $U, B, V$ and $R$ passbands as a function of the logarithm of the wavelength is roughly linear. The slope of this line is a good indicator of the companion if the color of the secondary star differs significantly from that of the Cepheid.

The values of the slope have been plotted in Figure 1 as a function of $\log P$. The programme stars have been divided into four groups:

- "small" amplitude Cepheids with known companion (circles),

- other small amplitude Cepheids (diamonds),

- "normal" amplitude Cepheids with known companion (asterisks),

- other normal amplitude Cepheids (squares).

The blue companions strongly move the slope towards zero. As to normal amplitude Cepheids, the smallest absolute values of the slope almost always correspond to Cepheids belonging to known binary systems having a blue companion. The exceptions can be those Cepheids where the companion has not been detected yet. The red 
companions are usually much less luminous, therefore their effect is not as obvious as that of a blue secondary.

On the basis of this method the following Cepheids certainly belong to binary systems (because the other method to be discussed later on also indicates a companion): VZ CMa, FM Cas, CR Cep, V402 Cyg, V1154 Cyg, V440 Per and DR Vel. Their duplicity has not been reported previously. In addition, several stars have a value of the slope as if they had a blue companion without any spectroscopic confirmations: SY Aur, YZ Aur, SS Sct and RY Vel.

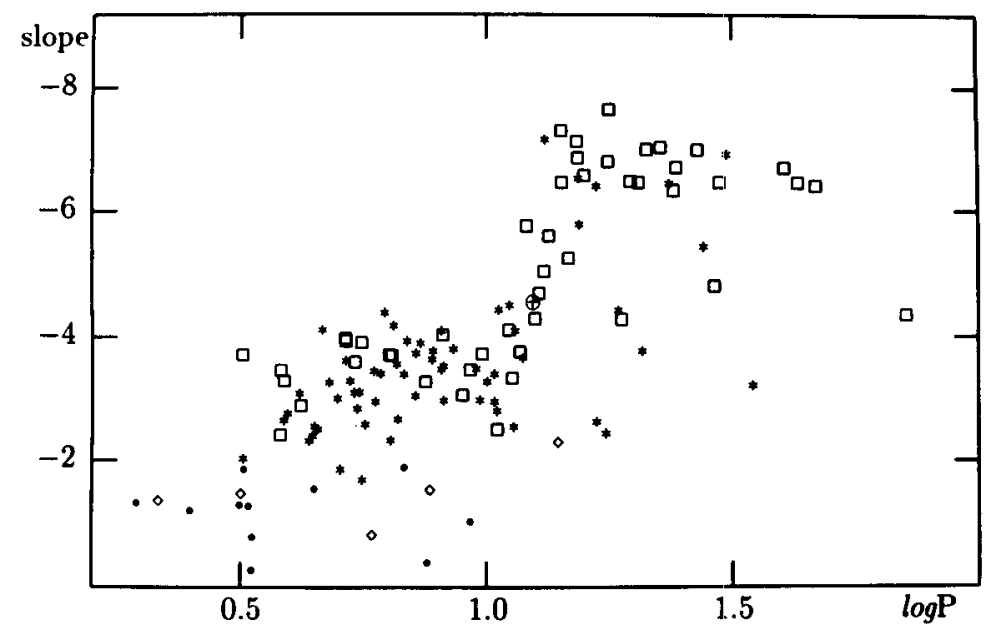

Figure 1 The period - slope relation.

\section{The AR-method}

The other method indicating the binary nature of the star makes use of both photometric and radial velocity data. The companion decreases the observable photometric amplitude but does not influence the observable pulsational radial velocity amplitude. As a consequence of this, the ratio of the amplitudes $A R=A_{\text {rad.vel. }} / A_{B}$ is larger for binary Cepheids. However, it cannot be assumed a priori that a single value of this amplitude ratio gives an adequate description for all Cepheids without companion.

The detailed study of the radial velocity amplitudes indicates that one cannot expect a single value of the amplitude ratio $A R$ for the solitary Cepheids but an unusually large value of $A R$ might well indicate the presence of a companion.

In addition to the seven stars newly classified as binary Cepheids (listed when discussing the "slope"-method), there are at least ten galactic Cepheids suspected in having a companion based on the large $A R$ value: V1344 Aql, ER Car, XY Cas, BD Cas, BY Cas, V381 Cen, V419 Cen, MY Pup, EV Sct, EU Tau, and the Magellanic Cloud Cepheids HV834, HV1365 and HV2864. A thorough spectroscopic study of these Cepheids would be worthwhile.

Travel grants from the OTKA (Hungary) and IAU are gratefully acknowledged. 